\title{
The Central Laser Facility at the Pierre Auger Observatory
}

\author{
F. Arqueros, J. Bellido, C. Covault, D. D’Urso, C. Di Giulio, P. Facal, B. Fick, F. Guarino, \\ M. Malek, J.A.J. Matthews, J. Matthews, R. Meyhandan, M. Monasor, M. Mostafa, \\ P. Petrinca, M. Roberts, P. Sommers, P. Travnicek, L. Valore, V. Verzi, L. Wiencke \\ for the Pierre Auger Collaboration. \\ Coordinator: (L. Wiencke wiencke@ cosmic.utah.edu usa-malek-M-abs1-he15-poster)
}

The Central Laser Facility is located near the middle of the Pierre Auger Observatory in Argentina. It features a UV laser and optics that direct a beam of calibrated pulsed light into the sky. Light scattered from this beam produces tracks in the Auger optical detectors which normally record nitrogen fluorescence tracks from cosmic ray air showers. The Central Laser Facility provides a "test beam" to investigate properties of the atmosphere and the fluorescence detectors. The laser can send light via optical fiber simultaneously to the nearest surface detector tank for hybrid timing analyses. We describe the facility and show some examples of its many uses.

\section{Introduction}

The southern Pierre Auger Observatory in Mendoza Province, Argentina measures extensive air-showers produced by cosmic rays. A surface detector (SD) records the distribution of charged particles at ground level. A fluorescence detector (FD), consisting of independent eyes operating at night, records light profiles of showers as they develop in the atmosphere. The FD makes a calorimetric measurement of shower energy because the amount of fluorescence light emitted is proportional to the energy deposited in the atmosphere.

The Central Laser Facility (CLF) (Fig. 1 provides a laser generated "test beam" for the observatory. The CLF tracks recorded by the FD eyes have several similarities to tracks generated by extensive air showers. The laser wavelength of $355 \mathrm{~nm}$ is near the middle of the air shower fluorescence spectrum. After accounting for atmospheric effects, the total amount of light scattered out of the beam is proportional to the laser beam energy. At full laser energy, the amount of light scattered out of the beam is roughly equivalent to the amount of fluorescence light emitted by showers with energy in region of sensitivity of the FD to the predicted GZK

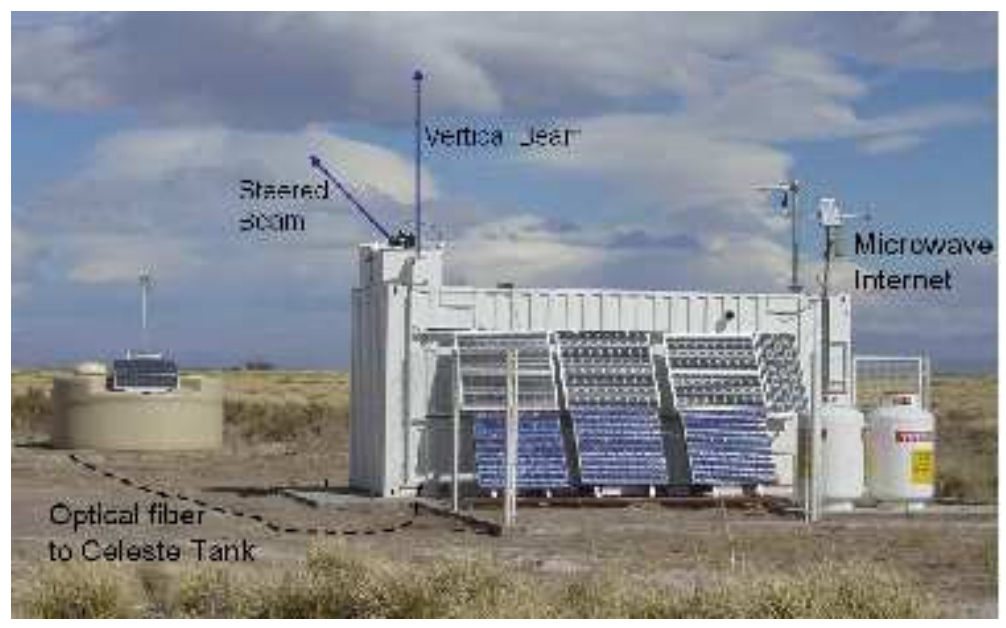

Figure 1. The Central Laser Facility at the Pierre Auger Southern Observatory. 


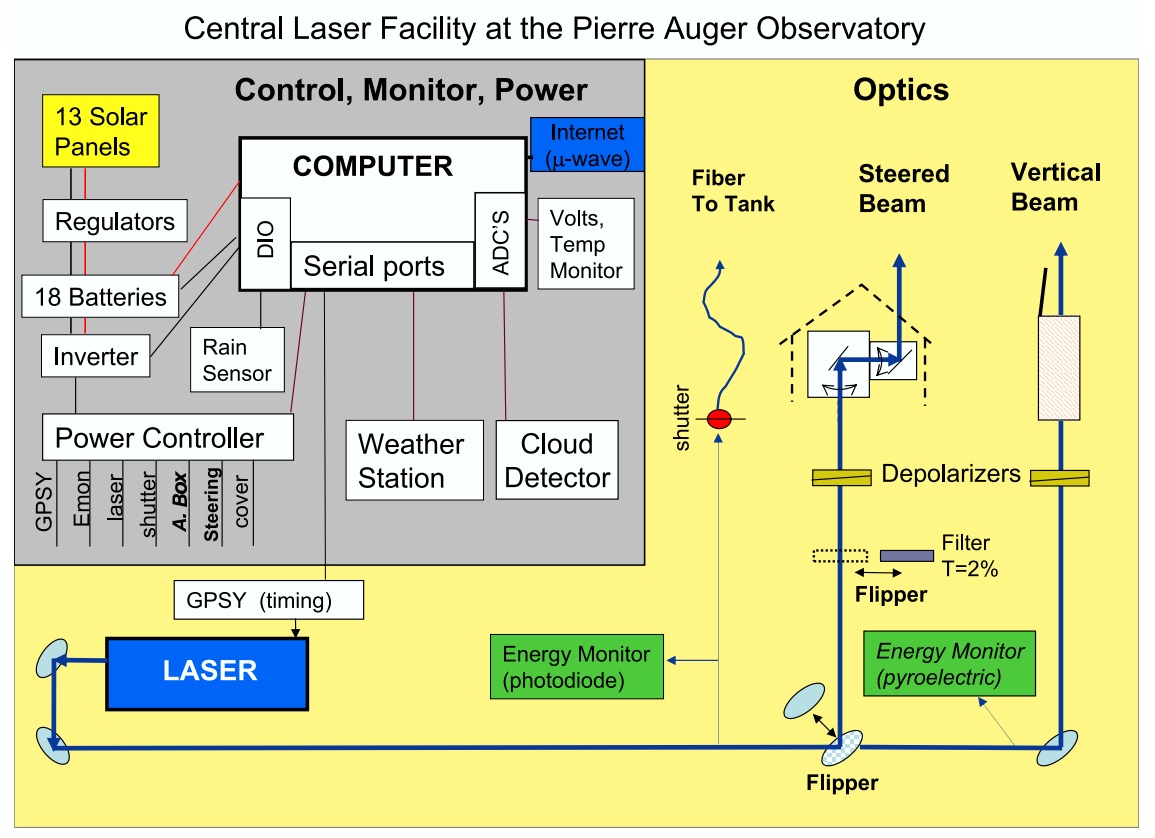

Figure 2. The major components of the CLF.

suppression. The CLF can direct light simultaneously into a SD tank via optical fiber to test the relative timing between the FD and SD. The facility also houses a weather station and a radiometric cloud detector [1].

\section{Hardware Description}

The heart of the system is a frequency tripled YAG laser producing a $7 \mathrm{~ns}$ pulsed beam with a maximum energy per pulse of $7 \mathrm{~mJ}$. The optical configuration (Fig. 2) includes two harmonic separator mirrors arranged so that the spectral purity of the $355 \mathrm{~nm}$ beam sent to the sky is better than $99.9 \%$. A "flip mirror" selects between two vertical beam paths. One goes directly to the sky when a simple cover is open. The other enters a mechanism with two mirrors on rotating orthogonal axes that can steer the beam in any direction above the horizon. A mechanical cover protects the steering apparatus when not in use. The redundancy of this dual beam configuration increases system reliability and simplifies operation.

The beams are calibrated and configured with randomized polarization. The symmetric scattering properties of a depolarized beam are desirable: in this case a vertical beam scatters light equally in the direction of each FD eye. Depolarizers in each beam path convolve the linearly polarized beam emitted by the laser to an output beam for which the net polarization is within $3 \%$ of random. The relative energy of each pulse is monitored by a photo-diode detector. A pyroelectic energy probe, installed in March 2005, makes a second relative measurement of the vertical beam energy. This probe, factory calibrated to NIST standards, is used to calibrate the photo-diode probe in terms of energy delivered to the sky. This procedure is performed several times per year. Energy and polarization calibrations are performed downstream of the last optical element before the beams enter the sky. 


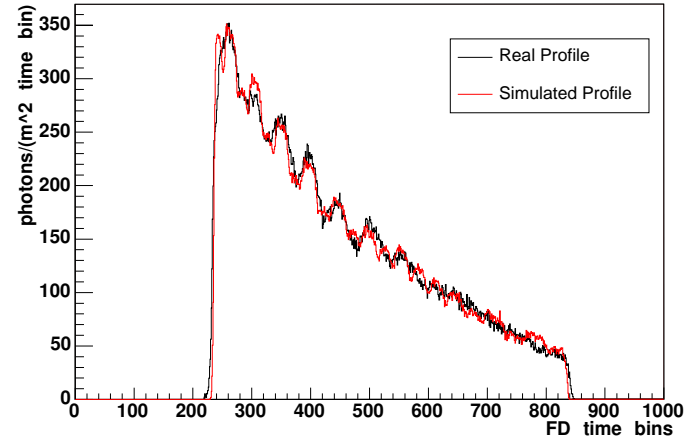

Figure 3. Simulated and measured FD detector response to a vertical CLF laser shot. The detailed simulation in this example includes a complete model of the detector optics, pixel elements, and reflective triangular inserts between pixels. One FD time bin is $100 \mathrm{~ns}$.

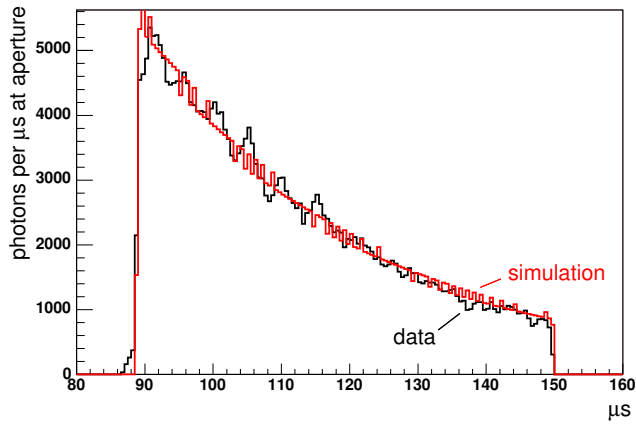

Figure 4. Light profile at the aperture of the Los Leones FD of a vertical CLF laser shot recorded under extremely clear atmospheric conditions. This simplified simulation assumes an aerosol-free atmosphere. The horizontal axis corresponds to the time since the laser fired.

The CLF is solar powered, housed in a modified shipping container, and operated remotely via microwave Internet link. The facility is unmanned. No wires run to the CLF. A small computer (PC) monitors the solar power system, collects weather station and cloud detector data, and operates the laser system. All of the control software is written using standard C programming tools. The PC which consumes 5 watts, runs a 25 MB version of embedded Linux, and has no moving parts. Each time the laser is fired, the time, relative energy, beam path ID, and direction are recorded to a compact flash card. All data are copied off-site daily.

The laser firing time relative to the GPS second is controlled by a programmable timing module [2]. Every shot is tagged to avoid confusion with possible neutrino-generated upward going tracks. A small fraction of the laser light can be injected into the nearest SD water tank (dubbed "Celeste") via optical fiber. The "sky-only" shots are recorded by each triggered FD to a special laser file. The "sky+Celeste" shots are written to the same data stream as air shower candidates together with the corresponding data from the Celeste tank.

Laser operation is conducted from the observatory's central campus by starting and stopping the control program at the beginning and end of each night of FD operation. Sets of 50 fixed-direction vertical shots are fired every 15 minutes followed by one sky+Celeste shot. Patterns of inclined shots are also fired during two onehour periods each night. This pattern include shots fired along the bisector angles between FDs and low-energy near-horizontal shots fired over each FD eye.

\section{Examples of Use}

On nights scheduled for FD operation, the observation of CLF tracks provides real-time confirmation that the FD eyes are functioning and are able to "see" the array center. Shots fired into the sky and the Celeste tank are used to monitor the relative timing between the FD and the SD [3]. A fit to the longitudinal profile of vertical tracks recorded by the FDs has been normalized to a clear air profile to generate an hourly data base of aerosol optical depth measurements [4]. CLF shots are used in ongoing studies of triggering efficiency, geometrical reconstruction [5] and the details of the FD pixel-pixel response (Fig. 3). Sweeps of inclined shots are also used to test the FD mirror pointing directions. Work in progress compares the profiles of CLF vertical tracks recorded under extremely clear atmospheric conditions to simulated profiles assuming an aerosol-free 

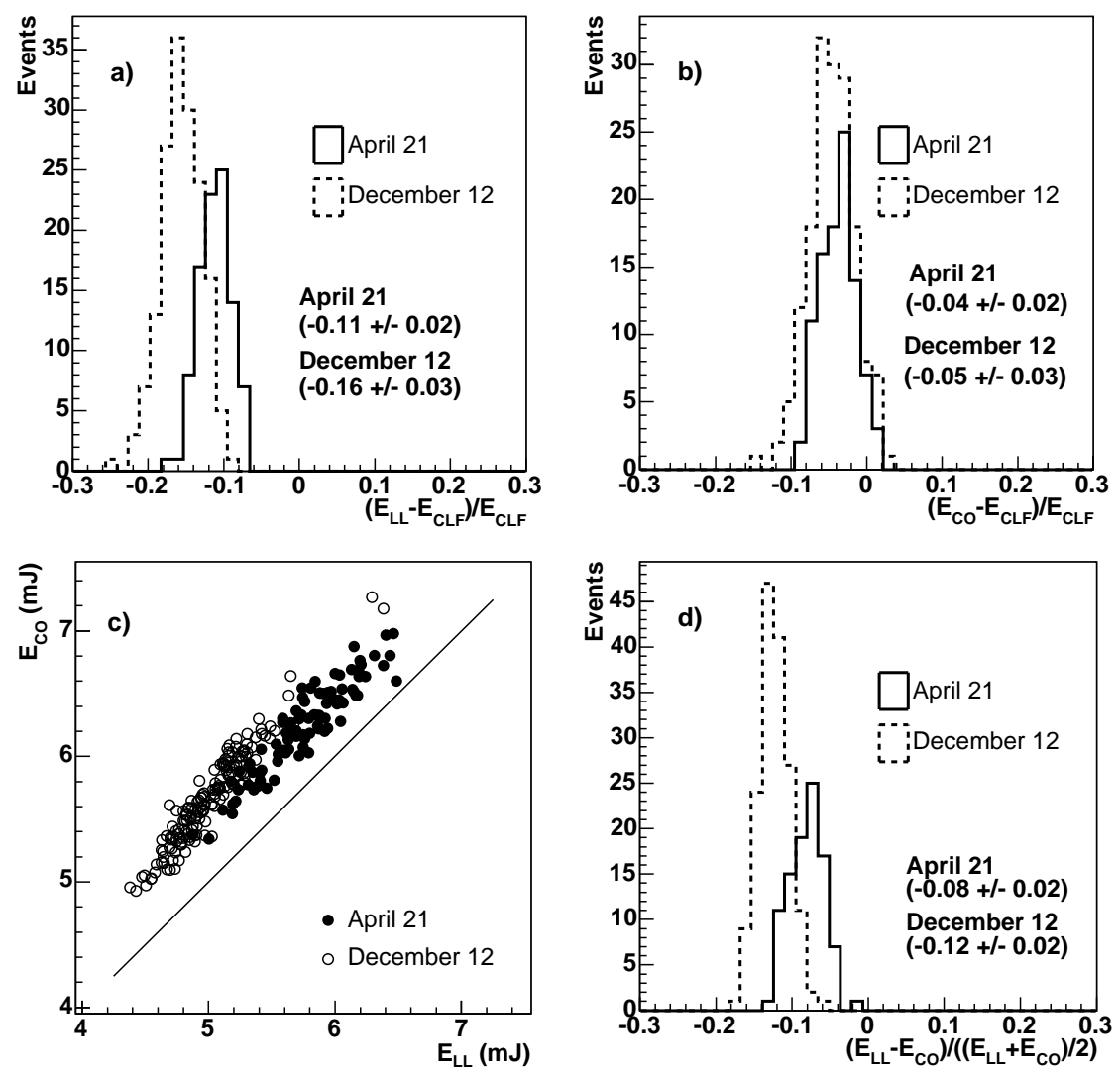

Figure 5. A vertical beam from the CLF is used in a global cross-check of photometric calibration for two nights with extremely clear viewing conditions. This cross-check involves two FD eyes, the CLF, the molecular atmospheric description, and $60 \mathrm{~km}$ of total light path. The consistency in the measured and reconstructed CLF energies is at the level of 15\% (a,b). The correlation in laser energy as reconstructed by the Coihueco (CO) and Los Leones (LL) eyes (c,d) demonstrates the sensitivity of this test. The results are consistent with the current level of uncertainty in the calibrations of the elements tested including the atmosphere.

atmospheric model. (Fig. 4). These comparisons are used to cross-check the calibration of the fluorescence telescopes (Fig. 5). This work tests, in combination, the photometric calibration of the CLF and two FD eyes, the identification of clear conditions, and modeling of molecular atmospheric conditions over a total light path distance of $60 \mathrm{~km}$.

\section{References}

[1] D. Riordan et al. J. Geophys. Res., 110, D03207, (2004).

[2] J.D. Smith, S. B. Thomas, L. R. Wiencke et al., Proc of 27th ICRC, HE175, (2001).

[3] P. Allison et al., Proc of 29th ICRC, HE 1.5 (2005).

[4] R. Cester et al., Proc of 29th ICRC, HE 1.5 (2005).

[5] J. Bellido et al., Proc of 29th ICRC, HE 1.5 (2005). 